\title{
Captura de Carbono en sistemas pastoriles establecidos en Colombia
}

\author{
Carbon sequestration in pastoral systems established in Colombia \\ Latriglia Castro Leslie Xiomara ${ }^{1}$ y Vera Oyola César ${ }^{2}$ \\ ${ }^{1}$ I.A. Universidad de los Llanos y \\ 2..A. Docente Universidad de los Llanos \\ cvera@unillanos.edu.co
}

Recibido 12 de Diciembre 2013, Aceptado 10 de Abril 2015

\section{RESUMEN}

En la zona de la altillanura Colombiana se ha observado un alto potencial para la recuperación de áreas degradadas, captura y almacenamiento de carbono $(C)$, puesto que existen grandes extensiones de sistemas pastoriles, por tanto se podría implementar proyectos que conlleven a la reducción de emisiones de gases de efecto invernadero (GEI), particularmente de dióxido de carbono $\left(\mathrm{CO}_{2}\right)$, lo cual podría ser similar al de algunos ecosistemas de bosque nativo secundario. Su difusión tiene como fin contribuir a la reflexión sobre los riesgos ambientales en que se podría incurrir al transformar un ecosistema natural de pastizales como los Llanos Orientales de Colombia. Inicialmente se realiza una descripción generalizada de lo que es la captura de carbono, ciclo de carbono, el cambio climático y gases de efecto invernadero, protocolo de Kyoto, sistema pastoriles con sumidero de carbono, parámetros que se determinan en la captura de carbono, producción primaria neta aérea y subterránea, la retención y acumulación de $C$ en suelos bajo pastos de gramíneas introducidas. También se realiza una identificación de las condiciones agroecológicas y socioeconómicas más relevantes de la altillanura colombiana, que aporten la mayor cantidad de elementos sobre la oferta que le brinda esta subregión a la captura de carbono, en factores como suelos, clima, infraestructuras y recursos humanos, realizando un análisis comparativo entre los requerimientos agroclimatológicos y la captura de carbono como sistema productivo, donde se establecen las principales oportunidades y fortalezas del tema. Se concluye que la generación de forrajes 
adaptados a suelos marginales es clave para maximizar los beneficios de las pasturas mejoradas, además de incrementar el secuestro de carbono las pasturas pueden ser parte sistemas agrosilvopastoriles, con efectos positivos en el rendimiento de los cultivos y la mitigación del efecto invernadero.

Palabras clave: Captura de carbono, gases de efecto invernadero, cambio climático.

\section{ABSTRACT}

In the area of the Colombian high plains it has witnessed a high potential for recovery of degraded areas, carbon capture and storage $(C)$, since there are large tracts of grazing systems therefore could implement projects that lead to reduction emissions of greenhouse gases (GHG), particularly carbon dioxide $\left(\mathrm{CO}_{2}\right)$, which may be similar to some secondary native forest ecosystems. Dissemination is to contribute to the reflection on the environmental risks that might be incurred to transform a natural grassland ecosystem and the eastern plains of Colombia. Initially a general description of what is carbon sequestration, the carbon cycle, climate change and greenhouse gases, Kyoto protocol, pastoral system with carbon sink parameters determined in carbon sequestration, production takes place Aerial and ground net primary, retention and accumulation of $C$ in soils under pastures of introduced grasses. Identification of the most important agro-ecological and socio-economic conditions of the Colombian high plains, which provide the largest number of items on offer that gives you the subregion to carbon sequestration in poor soils, climate, infrastructure and human resources is also made, performing a comparative analysis between the agroclimatological requirements and carbon capture and production system, where the main opportunities and strengths of the issue are set. It is concluded that the generation of fodder adapted to marginal soils is key to maximizing the benefits of improved pastures, and increase carbon sequestration pastures can be part agroforestry systems, with positive effects on crop yields and mitigation greenhouse effect.

Keywords: Carbon capture, greenhouse gases, climate change. 


\section{RESUMO}

$\mathrm{Na}$ área das altas planícies colombianas que tem testemunhado um grande potencial para recuperação de áreas degradadas, de captura e armazenamento de carbono (C), uma vez que existem grandes extensões de sistemas de pastejo, portanto, poderia implementar projetos que levam à redução as emissões de gases de efeito estufa (GEE), em particular o dióxido de carbono (CO2), que pode ser semelhante a alguns ecossistemas de florestas nativas secundárias. Divulgação é contribuir para a reflexão sobre os riscos ambientais que possam ser incorridos para transformar um ecossistema pastagem natural e as planícies orientais da Colômbia. Inicialmente uma descrição geral do que é o seqüestro de carbono, o ciclo do carbono, as alterações climáticas e gases de efeito estufa, o protocolo de Kyoto, sistema pastoral com os parâmetros de sumidouros de carbono determinadas no sequestro de carbono, a produção tem lugar aérea e terrestre rede primária, retenção e acúmulo de $\mathrm{C}$ em solos sob pastagens de gramíneas introduzidas. Identificação das condições agro-ecológicas e socioeconómicas mais importantes das altas planícies colombianas, que fornecem o maior número de itens em oferta que lhe dá a sub-região para o sequestro de carbono em solos pobres, clima, infra-estrutura e recursos humanos também é feito , realizando uma análise comparativa entre os requisitos agroclimatológicas e captura de carbono e sistema de produção, onde as principais oportunidades e pontos fortes do problema estão definidos. Conclui-se que a geração de forrageiras adaptadas a solos marginais é fundamental para maximizar os benefícios de pastagens melhoradas, e aumentar pastagens de seqüestro de carbono pode ser sistemas agroflorestais parte, com efeitos positivos sobre a produtividade das culturas e mitigação efeito estufa.

Palavras-chave: captura de carbono, gases de efeito estufa, mudança climática.

\section{INTRODUCCIÓN}

En el ámbito mundial existe preocupación por el calentamiento de la tierra, debido a las emisiones de gases causantes del efecto invernadero, donde el dióxido de 
carbono $\left(\mathrm{CO}_{2}\right)$ es el que más contribuye y el $64 \%$ de este gas es generado por el hombre, lo que demuestra su gran responsabilidad en el calentamiento global. En los últimos 150 años la concentración de $\mathrm{CO}_{2}$ ha subido un $30 \%$ y se estima que la mayoría del aumento de las concentraciones atmosféricas proviene del uso de combustible fósiles, mientras que el $20-25 \%$ se da por el cambio del uso de la tierra (IPPC, 2000).

En América Latina durante las últimas décadas del siglo $X X$, los ecosistemas forestales fueron talados en alta proporción, para promover el pastoreo de ganado. A escala global desde 1950, 200 millones de hectáreas de bosque húmedo y su biodiversidad asociada se han perdido, aparentemente teniendo como causa principal, la expansión de la ganadería (FAO, 2001; Szott et al., 2000). Ciesla (1996) manifiesta que estos sistemas con pasturas tradicionales mal manejadas son grandes aportantes de gases de efecto invernadero (dióxido de carbono, metano y óxido nitroso) a la atmósfera.

Los sistemas agroforestales y entre ellos los silvopastoriles, son una opción apropiada para enfrentar la problemática, porque tienen el potencial de conservar los recursos naturales y la biodiversidad controlando la erosión de los suelos; por ejemplo, el carbono puede acumularse en pastos en rotación o en sistemas pastoriles (Thornley et al., 1991). Las praderas naturales son un importante reservorio para el carbono, porque los gases contribuyen a la acumulación de materia orgánica del suelo (MOS) (Martínez et al., 2008). Se ha demostrado que a diferencia de las gramíneas nativas, las especies africanas introducidas en las sabanas de Sudamérica incrementan la MOS y acumulan carbono en el suelo (Fisher et al., 1998). Además, la mayoría de las praderas nativas o sabanas en el trópico son frecuentemente quemadas (anualmente) y poco fertilizadas. Hay autores que sugirieren que el nivel actual del carbono en el suelo puede incrementarse si cesan las quemas y se aplica algún fertilizante, es posible que con estas simples opciones de manejo, estos pastizales tropicales, sean un reservorio mayor de carbono que lo que actualmente se pronostica (Mendieta y Rocha, 2007). 
Casanova et al., (2011) indican que varios factores favorecen la retención del carbono en el suelo y permiten mayores tasas de recambio y tiempos de residencia, estos incluyen, distribución por debajo de la superficie del suelo, asimilados con bajo contenido de nutrimentos, materiales ricos en lignina y ceras, inundación, bajas temperaturas, texturas arcillosas, alta saturación de bases, agregación y superficies de cargas variables. La magnitud con la cual el suelo puede ser un sumidero de carbono depende del balance entre las tasas de los procesos de adquisición y la tasa de rotura tanto de carbono residente como de carbono adquirido (FAO, 2000).

Un buen sistema agropecuario es el que secuestra más carbono del que emite (Mora, 2001), las pasturas con base en gramíneas mejoradas lo secuestran en mayor cantidad en partes profundas del perfil del suelo, generalmente debajo de la capa arable $(10-15 \mathrm{~cm})$, esta característica hace que el carbono esté menos expuesto a los procesos de oxidación y por lo tanto reducir su pérdida como gas invernadero (Fisher et al., 1998).

\section{EL CICLO DEL CARBONO}

El carbono, elemento principal de las biomoléculas, se encuentra presente en la atmósfera en forma de $\mathrm{CO}_{2}$, en la hidrosfera como bicarbonato y como ion carbonato, y en la litosfera en forma de rocas carbonatadas, carbón mineral o petróleo. Mediante la fotosíntesis o la quimiosíntesis, los productores primarios incorporan el carbono de la atmósfera y la hidrosfera en forma de $\mathrm{CO}_{2}$ y lo integran en sus tejidos. A través de la cadena trófica marina y terrestre, los consumidores se alimentan de los productores y el carbono pasa a formar parte de todos los organismos vivos, que lo devuelven a la atmósfera con su respiración, o al subsuelo en forma de excrementos, o, tras su descomposición, generando rocas carbonatadas. La actividad volcánica y la utilización por el hombre de combustibles fósiles, como petróleo o carbón, devuelven también una gran cantidad de $\mathrm{CO}_{2}$ a la atmósfera (Arana, 2001). 
El ciclo biológico del carbono (Figura 1) es relativamente rápido: se estima que la renovación atmosférica ocurre cada 20 años. En ausencia de la influencia antropogénica, en el ciclo biológico existen tres depósitos o "stocks": terrestre (20000 Gigatones (Gt $=1 \times 10^{9}$ toneladas)), en atmósfera (750 Gt) y océanos (40000 Gt). Este ciclo desempeña un papel importante en los flujos de carbono entre los diversos depósitos, a través de los procesos de fotosíntesis y respiración, mediante el primer proceso, las plantas absorben la energía solar y el $\mathrm{CO}_{2}$ de la atmósfera, produciendo oxígeno e hidratos de carbono, que sirven de base para su crecimiento. Los animales y vegetales utilizan los carbohidratos en el proceso de respiración, usando su energía para diferentes reacciones metabólicas para su nutrición que genera $\mathrm{CO}_{2}$, que junto con la descomposición orgánica que es la forma de respiración de las bacterias y hongos, devuelven el carbono, biológicamente fijado en los reservorios terrestres que son: tejidos de biota, permafrost del suelo y turba, a la atmósfera (Gallardo y Merino, 2007). Las ecuaciones químicas que rigen estos dos procesos son:

- Fotosíntesis: $6 \mathrm{CO}_{2}+6 \mathrm{H}_{2}+$ energía (luz solar) $\rightarrow \mathrm{C}_{6} \mathrm{H}_{12} \mathrm{O}_{6}+6 \mathrm{O}_{2}$

- Respiración: $\mathrm{C}_{6} \mathrm{H}_{12} \mathrm{O}_{6}$ (materia orgánica) $+6 \mathrm{O}_{2} \rightarrow 6 \mathrm{CO}_{2}+6 \mathrm{H}_{2}+$ energía

Es posible verificar que el mayor cambio entre el depósito terrestre y el atmosférico resulta de los procesos de fotosíntesis y respiración. Los días de primavera y verano, las plantas absorben luz solar y $\mathrm{CO}_{2}$ de la atmósfera y, paralelamente, los animales, plantas y microorganismos, a través de la respiración, devuelven el $\mathrm{CO}_{2}$. Cuando la temperatura o la humedad son bastante bajas, por ejemplo en invierno o en los desiertos, la fotosíntesis y la respiración se reducen o cesa, así como el flujo de carbono entre la superficie terrestre y la atmósfera.

Debido a la declinación de la tierra y a la desigual distribución de la vegetación en los hemisferios, existe una flotación a lo largo del año que es visible en los diversos gráficos de variación de concentración anual del $\mathrm{CO}_{2}$. En 1958, el científico Charles David Keeling (oceanógrafo del Scripps Institute of Oceanography), puso en marcha una serie de experiencias en el monte Mauna 
Loa, Hawaii, que le permitieron medir, con bastante precisión, la concentración de $\mathrm{CO}_{2}$ en la atmósfera.

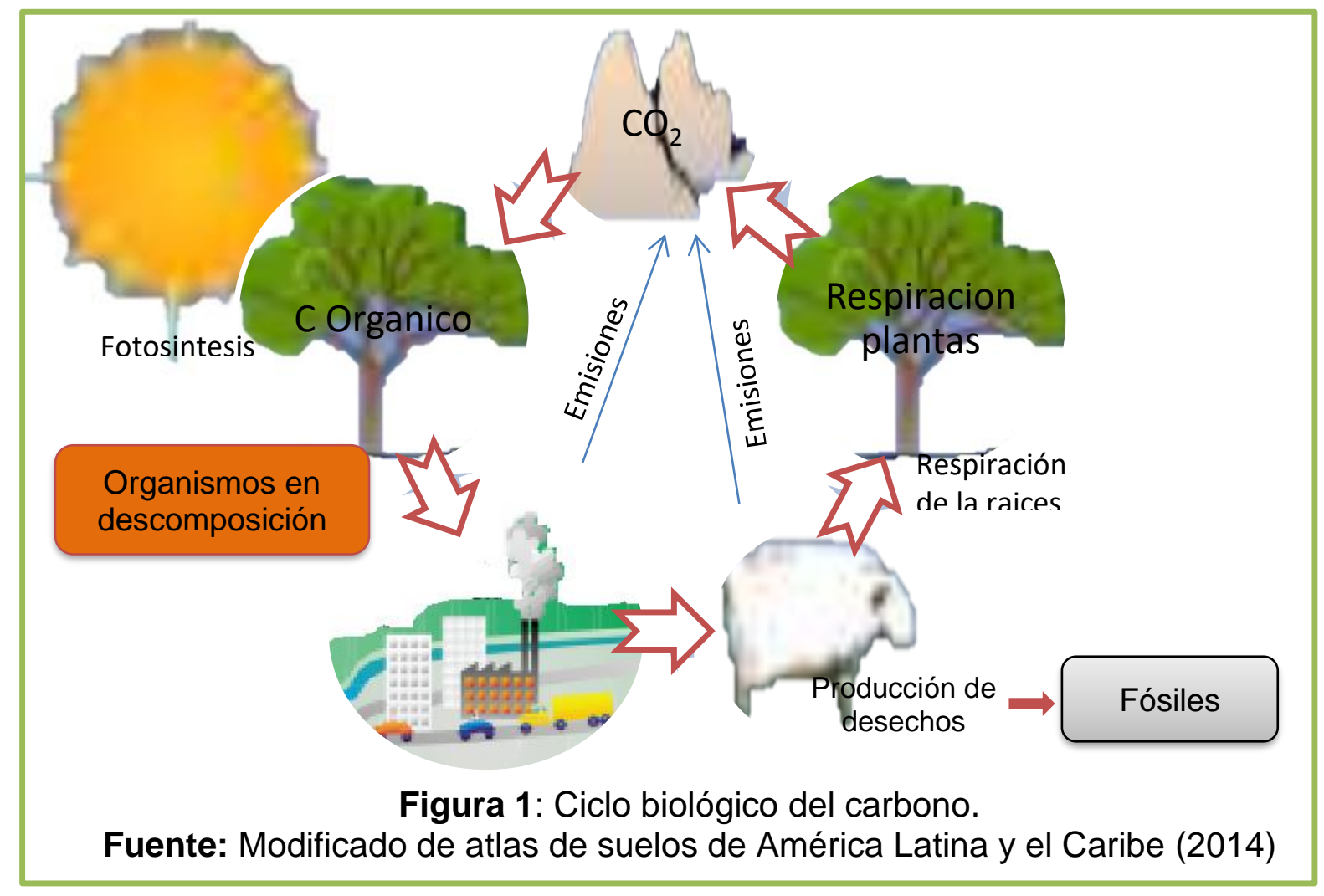

A pesar de que el reservorio atmosférico de carbono es el menor, cerca de $750 \mathrm{Gt}$, en comparación a los otros dos (océanos y terrestre), este depósito influye en el clima terrestre (Figura 2). Además, los flujos anuales entre la reserva atmosférica y las otras dos reservas mencionadas anteriormente, son muy sensibles a los cambios, siendo mayores los depósitos en los océanos, cincuenta veces mayor que la reversa atmosférica, además existen traspasos entre estos dos últimos stocks que a través de procesos químicos establecen un equilibrio de las concentraciones de $\mathrm{CO}_{2}$ entre las capas superficiales de los océanos y las del aire, dependiendo su absorción de la temperatura y la cantidad de $\mathrm{CO}_{2}$, cuando la superficie del océano tiene bajas temperaturas potencian su absorción atmosférica, mientras que las cálidas pueden causar la emisión de $\mathrm{CO}_{2}$ (Keeling, 1978; Lefévre et al., 2004). 


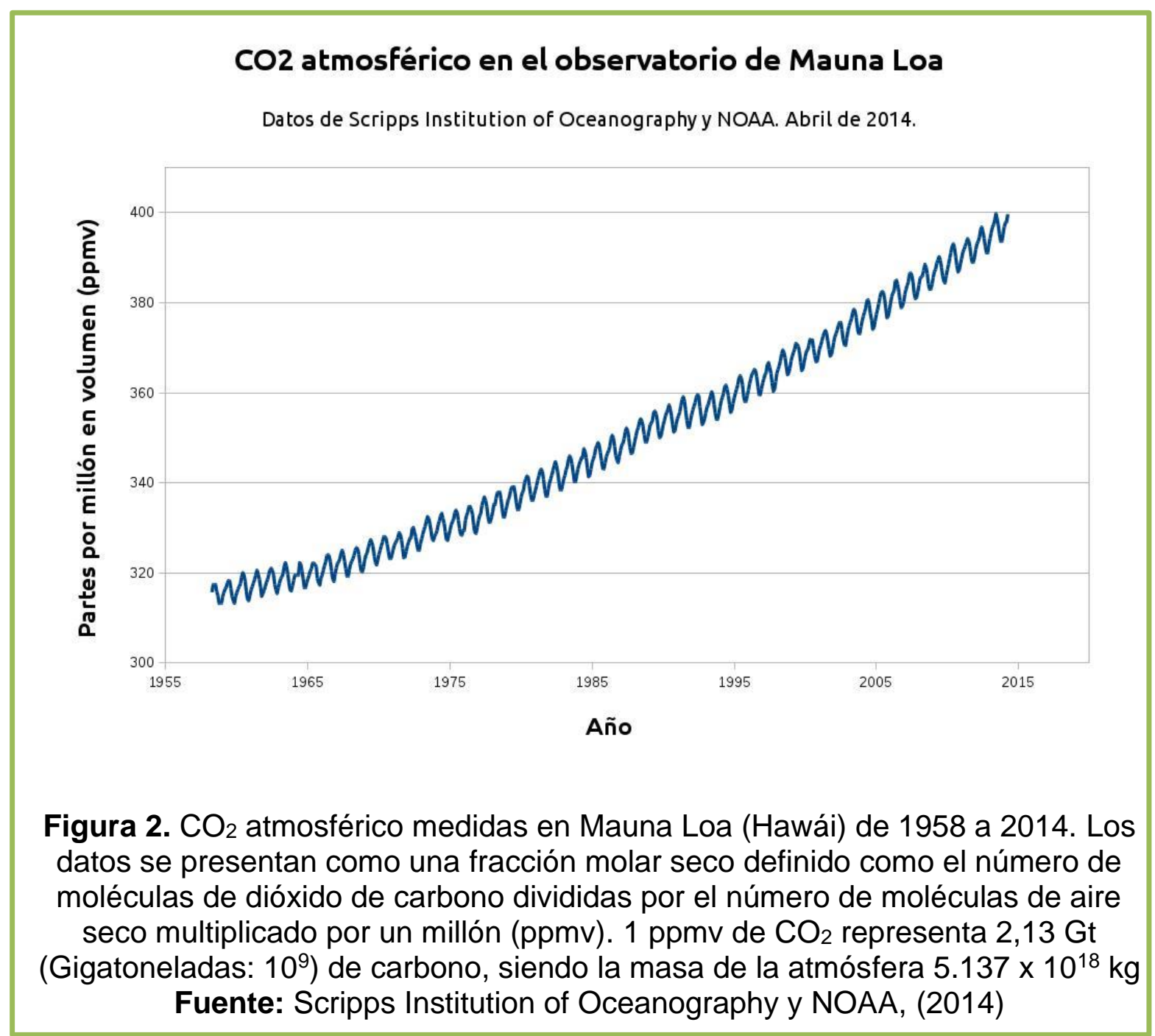

El flujo de carbono entre su depósito y liberación, sin la intervención del hombre, es similar pero con una lenta variación hacia el primero, se estima que en estas condiciones cada 20 años hay una renovación total del carbono atmosférico (Bravo, 2011), donde la vida en los océanos consume grandes cantidades de $\mathrm{CO}_{2}$, pero el ciclo entre la fotosíntesis y la respiración se desarrolla a una velocidad mayor. El fitoplancton es consumido por el zooplancton en sólo algunos días, y pequeñas cantidades de carbono son acumuladas en el fondo del mar, lo cual sucede cuando las conchas del zooplancton, compuestas de carbonato de calcio, se depositan en el fondo tras su muerte, se estima que el fitoplancton marino fija 45 Gt de $\mathrm{C}$ orgánico al año, de las cuales 16 son exportadas al interior del océano 
(Falkowski et al., 1998); otro proceso intermedio del ciclo biológico que provoca su remoción, ocurre cuando la fotosíntesis excede la respiración y, lentamente, la materia orgánica forma depósitos de sedimentos que, en ausencia de oxígeno y después de millones de años, se transforman en combustibles fósiles. Por último, los incendios son otro elemento del ciclo rápido que añaden $\mathrm{CO}_{2}$ a la atmósfera al consumir la biomasa y materia orgánica, y al provocar la muerte de plantas que acaban por descomponerse y formar también $\mathrm{CO}_{2}$ (Arana, 2001).

\section{EL CARBONO EN ECOSISTEMAS}

El $\mathrm{CO}_{2}$ atmosférico es incorporado a los procesos metabólicos de las plantas mediante la fotosíntesis, donde participa en la composición de todas las estructuras necesarias para que la planta pueda desarrollarse (follaje, ramas, raíces y tronco), es por esto que los agroecosistemas acumulan carbono en cuatro grandes componentes: biomasa aérea (sobre el suelo), hojarasca, sistema radical y carbono orgánico del suelo. Durante el tiempo en que el $\mathrm{CO}_{2}$ se encuentra constituyendo alguna estructura del árbol y hasta que es enviado nuevamente al suelo o a la atmósfera, se considera almacenado. En el momento de su liberación (ya sea por la descomposición de la materia orgánica y/o por la quema de la biomasa) el $\mathrm{CO}_{2}$ fluye para regresar al ciclo del carbono (Ortiz y Riascos, 2006).

Entre los métodos empleados para determinar la cantidad de $\mathrm{CO}_{2}$, se encuentra el factor de conversión o fracción de carbono que al multiplicarlo por la biomasa total involucra la relación entre el peso de la molécula de $\mathrm{CO}_{2}$ y el peso del átomo de carbono. Este factor generalmente tiene un valor de $0.5 \mathrm{gr}$ de $\mathrm{C}$ con respecto a 1 gr de biomasa (Ortiz y Riascos, 2006).

Carbono almacenado (Ca): Hace referencia a la cantidad de carbono que se encuentra en un ecosistema vegetal, en un determinado momento, tiene en cuenta criterios como tipo de bosque o vegetación, densidad de la madera, factores de ajuste que se basan en datos de biomasa calculada a partir de volúmenes por hectárea de inventarios forestales. Generalmente, este tipo de carbono almacenado se expresa en toneladas por hectárea (ton/ha), y se paga por 
conservación de bosques y no puede ser liberado a la atmosfera, si se accede a un pago por servicios ambientales (Ortiz y Riascos, 2006)

Carbono fijado (Cf): Se refiere al flujo de carbono dentro de una unidad de área cubierta con vegetación en un lapso de tiempo. Su cuantificación permite predecir el comportamiento del carbono en cualquier momento durante el crecimiento de la población, lo que depende de las características de la especie, la tasa de crecimiento y la longevidad, así como de las condiciones del sitio: clima y rotación. Se expresa en toneladas de $\mathrm{C}$ por hectárea al año (ton C/ha/año) (Ortiz y Riascos, 2006).

\section{CAMBIO CLIMÁTICO GLOBAL Y GASES DE EFECTO INVERNADERO (GEI)}

El calentamiento global, es un proceso de origen antrópico, que resulta de la aceleración de un efecto natural del planeta (García, 2003), su importancia radica en las consecuencias catastróficas que está generando en todo el mundo, como el descongelamiento de los polos, incremento del nivel de mar, sequías, huracanes, tormentas, desplazamiento de áreas agrícolas, migración de enfermedades, extinción de especies, entre otras (SEMARNAT, 2009).

El efecto invernadero, es un fenómeno natural que ha permitido mantener la temperatura de la tierra en niveles constantes y apropiados, el cual ocurre, cuando la radiación solar visible penetra hasta la superficie de la tierra y la calienta, y ésta a su vez emite radiación térmica, parte de la cual es retenida por los denominados gases de efecto invernadero (GEI) entre los que se destacan: el dióxido de carbono, el metano, los clorofluorocarbonos y el óxido nitroso (Davila, 2011).

Un sistema climático es estable cuando la radiación solar absorbida está en equilibrio con la emitida por la tierra hacia el espacio, pero cuando la concentración de GEI en la atmósfera aumenta, esta armonía se rompe provocando un aumento artificial en la temperatura media global del planeta. Se estima que el GEl que más influye en el calentamiento climático mundial, es el dióxido de carbono $\left(\mathrm{CO}_{2}\right)$ y que la mayor parte de su incremento en las concentraciones atmosféricas, provienen del uso de combustibles fósiles, mientras 
que el $20-25 \%$ es generado por la agricultura, explotación comercial de bosques y aumento de la oxidación de materia orgánica en los suelos (Davila, 2011).

Entre los países desarrollados que contribuyen con la mayor parte con la emisión de gases de efecto invernadero, se encuentran: Estados Unidos (EE.UU.) con un $33 \%$ del total mundial y Rusia con un $17.4 \%$, también producen altos índices Brasil, China, India, Japón, Alemania, Reino Unido, Indonesia y Francia (Ortiz y Riascos, 2006).

Según el tercer informe del grupo I del Panel Intergubernamental sobre Cambio Climático (IPCC sus siglas en ingles), desde finales del siglo XIX la temperatura media de la superficie terrestre ha aumentado $0.6^{\circ} \mathrm{C}$ y las temperaturas mínimas tienden a duplicarse por década en comparación con las máximas $\left(0.2\right.$ y $0.1^{\circ} \mathrm{C}$, respectivamente). La superficie de los hielos en el hemisferio norte desde finales de los años 60 , se ha reducido entre un $10-15 \%$, así como también se ha observado un aumento ligero (uno a dos centímetros por década) del nivel del mar a lo largo del siglo XX (IPCC, 2002).

\section{PROTOCOLO DE KYOTO Y MERCADO DE CARBONO}

El Protocolo de Kyoto ( $\mathrm{PK}$ ) es el instrumento legal a través del cual se regulan límites y reducciones de emisiones de GEl, vinculadas a los países industrializados con obligaciones ante la Convención Marco de las Naciones Unidas sobre el Cambio Climático (UNFCCC sus siglas en inglés). Los países industrializados se comprometieron en Kyoto a reducir sus emisiones de GEI en al menos un 5.2\% con respecto al nivel de emisiones de 1990 y a evaluar resultados durante el período 2008-2013. En el PK también se establecieron los mecanismos que facilitarían el cumplimiento de las reducciones de emisiones de GEI en los países industrializados de un modo costo-efectivo propuesto por la ONU, (1998): 1) Comercio de emisiones (CE), 2) Implementación conjunta (IC) y 3) Mecanismo de desarrollo limpio (MDL).

Comercio de emisiones (CE): Este mecanismo permite la transferencia de reducciones de carbono "no usadas", entre países industrializados que tengan sus 
derechos de emisión por debajo de los límites permitidos, con aquellos que han excedido sus niveles en un período de cumplimiento dado, las unidades de venta se denominan Assigned Amount Units (AAU sus siglas en inglés) (Rojas, 2011).

Implementación conjunta (IC): Como su nombre lo indica, este mecanismo permite la disminución de gases de carbono de manera conjunta entre los países participantes. En este caso, se acreditan unidades de reducción de emisiones a favor del o los países que financian proyectos de mitigación de cambio climático, sus unidades de venta se denominan Emission Reduction Units (ERU sus siglas en inglés). Un país desarrollado que tenga emisiones que superen los límites previstos por el Protocolo, puede asociarse con otro desarrollado para que en ese período cuente con emisiones por debajo de los límites, no superando los niveles sumados de ambos países (ONU, 1998).

Mecanismo de desarrollo limpio (MDL): Permite que proyectos encaminados a fijar, reducir o evitar emisiones de gases de efecto invernadero en países en desarrollo, obtengan beneficios económicos adicionales a través de la venta de Certificados de Emisiones Reducidas (CER's). Este es el único de los tres mecanismos al que pueden acceder estos países, entre ellos, los de América Latina. Este Protocolo entró en vigencia en Febrero de 2005, después de la ratificación de Rusia en Septiembre de 2004, con un total de 127 países que suman el $61.6 \%$ de la emisión global. Con la aprobación del PK, en la tercera conferencia de las partes en 1997, se sentaron las bases para desarrollar el mercado del carbono a nivel internacional (Rojas, 2011).

Este mercado, es un conjunto de transacciones donde se intercambian cantidades de reducciones de emisiones de gases de efecto invernadero. Según Eguren, (2004) América Latina se ha convertido en la región de países en desarrollo más activa en este mercado emergente, con alrededor de US\$210.6 millones de créditos de carbono en negociación en el marco del MDL, basada en la convicción de que puede significar una herramienta útil para promover el desarrollo sostenible de la región. Aunque el valor de estos certificados aún no es claro en este mercado, se pueden distinguir dos grandes escenarios en donde se están 
negociando, uno definido por las transacciones de carbono que buscan cumplir con el marco establecido por el Protocolo de Kyoto y otro, por las iniciativas paralelas de comercio fuera del protocolo, como son las iniciativas voluntarias de restricción de emisiones y las decisiones federales y estatales de EE.UU. para mitigar GEI, país que no hace parte del Protocolo de Kyoto (Eguren, 2004).

\section{SISTEMAS PASTORILES SUMIDERO DE CARBONO}

Las zonas de pastoreo están incluidas en el Artículo 3.4 del protocolo de Kyoto y, como los bosques, juegan un papel importante en el secuestro de carbono, en primer lugar, según la FAO, (2002) ocupan 3.200 millones de hectáreas y almacenan entre 200 y 420 ton de carbono en el ecosistema total, gran parte debajo de la superficie y en un estado relativamente estable. El carbono del suelo en pastizales es estimado en 70 ton/ha, cifra similar al de suelos forestales. Se debe tener en cuenta que muchas áreas de pastoreo en las zonas tropicales y áridas son mal manejadas y están degradadas; y por lo tanto, ofrecen varias posibilidades de secuestro de carbono tanto en la incorporación a la estructura química de la biomasa aérea, como en la hojarasca, o en el sistema radicular y materia orgánica del suelo (Céspedes et al., 2012).

\section{DIFERENCIAS ENTRE LAS ESPECIES DE GRAMÍNEAS TROPICALES}

Pastos Brachiaria decumbens cerca a Brasilia han mostrado poca o ninguna acumulación de carbono, mientras que $B$. decumbens solo y en asociación con la leguminosa Pueraria phaseoloides en Carimagua han almacenado adicionalmente 25.6 y 34.1 ton/ha de carbono respectivamente (Fisher et al., 1998). Especulando sobre el porqué de las diferencias, Thomas y Asakawa, (1993) sugieren que la calidad de la hojarasca que afecta las tasas y patrones de descomposición podría ser la responsable esto. El material de $B$. decumbens tiene una proporción de $C: N$ de 88 comparado con 130, 126 y 117 de $A$. gayanus, $B$. dictyoneura y $B$. humidicola, respectivamente. Por otro lado, esta proporción en las raíces varía entre 159 y 224, además las interacciones entre el clima y el suelo también pueden ser otros factores que influye en estas diferencias. 


\section{ZONAS DE CULTIVO}

El desarrollo de la agricultura ha implicado una gran pérdida de materia orgánica del suelo, hay diferentes prácticas de manejo que pueden ser usadas para aumentar el contenido de la materia orgánica del suelo tales como: incremento de productividad y biomasa, variedades, fertilización e irrigación. Las fuentes de materia orgánica también incluyen residuos, compost y cultivos de cobertura. Las principales formas de obtener un incremento de materia orgánica en suelo, son las que están asociadas a la agricultura de conservación, labranza mínima o cero y el uso de una cobertura vegetal continua y protectora formada por materiales vegetales vivos o muertos sobre la superficie del suelo (FAO, 2002).

Las praderas ocupan alrededor de un quinto del área terrestre mundial, cerca de 3.4 billones de hectáreas (B ha) (FAO, 1993), principalmente están en áreas que han sido clasificadas en términos del potencial para el uso del suelo entre bajo 0 cero, cerca de $1.5 \mathrm{~B}$ ha o $44 \%$ de las praderas están en el trópico y cerca de la mitad (0.7 B ha) se consideran praderas mejorables (PNUMA, 2012).

Houghton, (1995) estimó que el contenido de carbono en la vegetación y el suelo en las praderas tropicales era de 16 y 48 ton/ha respectivamente, mientras que Fisher et al., (1998) consideraron que los valores de Houghton, (1995) eran 1.5 y 5 veces menos que las cantidades de carbono que ellos midieron en el suelo a un metro de profundidad, en los Llanos Orientales de Colombia.

Las sabanas, los bosques y las praderas tropicales ocupan al menos un $11.5 \%$ de la superficie terrestre total, la fijación neta de carbono gramínea-árbol es cerca de 7.6 petagramos al año ( $\mathrm{Pg} / \mathrm{año} ; 1 \mathrm{Pg}=$ mil millones de toneladas), aproximadamente la mitad de la acumulada anualmente en bosques, lo que es principalmente controlado por la disponibilidad de agua, nutrientes, composición y estructura de la vegetación (PNUMA, 2012). El almacenamiento total en las praderas, sabanas y bosques tropicales es cerca de $135 \mathrm{Pg}$, del cual el $80 \%$ está en el suelo (Amézquita et al., 2013). 


\section{AUMENTO DEL SECUESTRO DE CARBONO}

Existen varias formas para implementar el secuestro de carbono, en las que se considera los componentes del suelo, planta y los sistemas de manejo; entre otras alternativas a continuación se relaciona cinco opciones:

\section{Introducción de leguminosas forrajeras para mejorar el suministro de nitrógeno.}

Fisher et al., (1998) indicaron que la cantidad de carbono acumulado en pastos de gramíneas solas era marcadamente constante en 3 ton ha/año y sugirieron que el proceso era limitado en su tasa de incremento, como la adición de un componente leguminoso al pasto la elevaba de 2.5 hasta casi 5 veces, concluyeron que el proceso se limitaba por deficiencia de nitrógeno, sin embargo, si hay un valor de equilibrio para la máxima cantidad de carbono que un suelo puede secuestrar (Davidson et al., 1995), significa que para aumentar la tasa de acumulación de carbono, este valor se debe alcanzar rápidamente, pero aún no se sabe cuál podría ser esa cifra, ni qué mecanismos controla el proceso de aumento en el almacenamiento de carbono. Los mismos argumentos podrían ser ciertos para otras opciones que aumenten el suministro de nitrógeno en los pastos puros de gramíneas, como por ejemplo, modestas aplicaciones de fertilizantes nitrogenados o la búsqueda de medios para incrementar la fijación asociativa de nitrógeno (Fisher et al., 1998).

\section{Suelos mal drenados}

Los suelos en estas condiciones tienen un alto contenido de materia orgánica en comparación con los bien drenados, existen grandes áreas de este tipo de suelos en las sabanas de Colombia y Venezuela, pero no hay posibilidades económicas para mejorar sus drenajes, puesto que son inundados durante gran parte de la estación lluviosa. Si se logran encontrar gramíneas y leguminosas tropicales que puedan adaptarse al exceso de humedad, la productividad neta de estos suelos podría aumentar, como también contribuir sustancialmente al secuestro de carbono (FAO, 2000; CORPOICA, 2014). 


\section{Macrofauna del suelo}

Los pastos usualmente tienen mayor población de invertebrados comparados con otras formas de uso del suelo (Lavelle et al., 1994). En la altillanura colombiana, los pastos introducidos de gramíneas en monocultivo y en asociación con leguminosas tienen cinco y diez veces más peso vivo, proveniente de la macrofauna del suelo, hasta $60 \mathrm{~g} / \mathrm{m}^{2}$, (Decaens et al., 1995) que la sabana nativa. Las lombrices de tierra dominan la fauna del suelo y pueden ingerir diariamente diez veces el peso de su cuerpo. A partir de esto, Fisher et al., (1998) concluyeron que en tres años una masa de $60 \mathrm{~g} \mathrm{~m}^{2}$ tiene el potencial para pasar el volumen total de suelo en $0.5 \mathrm{~m}$ de profundidad.

Las poblaciones de lombrices de tierra deben tener un considerable impacto en los procesos de movimiento de carbono desde la superficie hasta mayores profundidades, aunque no se sabe si ellas son el principal vehículo o si existen otros procesos involucrados, se sabe que los excrementos de las lombrices de tierra tienen propiedades diferentes a las del suelo, como mayor estabilidad de agregados debido a la cementación de las partículas (Gugenberger et al., 1996). Tampoco se conoce el costo de carbono para mantener una población grande de macrofauna, ni los beneficios que ellas traen en términos de mejoramiento del suelo.

\section{Manejo del pastoreo}

Los datos sobre la influencia del manejo del pastoreo sobre la acumulación del carbono orgánico del suelo (COS) son inconsistentes (Manley et al., 1995), puesto que hay pocos datos porque la mayoría de los estudios de pastoreo se han enfocado en la producción de forraje y uso en la alimentación animal, con poca atención en el impacto sobre el recurso suelo (Fisher et al., 1998). Es claro que las prácticas de manejo que conllevan a una degradación del pasto reducen el COS, por lo tanto se necesita indicadores para integrar los factores involucrados en la respuesta de la materia orgánica del suelo (MOS) al pastoreo: tipo de suelo, estado fisiológico del pasto y nivel de nutrientes, un buen ejemplo de esto pasto 
desarrollado puede mejorar el crecimiento, productividad y senescencia en términos de una simple medición de altura en el pasto (Hodgson, 1990). Para optimizar la acumulación neta de COS la determinación del estado fisiológico del pasto puede unirse al concepto de Spain et al., (1995) que definen manejo de los forrajes debe darse en un "pastoreo flexible" porque puede asegurar la sostenibilidad de la producción, ya se están dando los primeros en pastos tropicales lo que requiere de mucha más investigación (Fisher et al., 1998).

\section{Manejo de fertilizantes}

Aunque fuera factible la aplicación de fertilizantes nitrogenados a los pastos tropicales para asegurar su sostenibilidad, existe un costo de carbono en la elaboración de la mayoría de ellos, si bien el uso de estos en pastos establecidos en las sabanas neotropicales es muy limitado, en sistemas agropastoriles mixtos las gramíneas hacen uso del fertilizante residual aplicado a los cultivos, los cuales necesitan mayores niveles de fertilidad (Faria, 2006). En contexto, es el cultivo -no el pasto- el que financia el costo económico y ambiental, por lo tanto, en sistemas agropastoriles, el uso de fertilizantes para aumentar la producción agrícola y la acumulación de carbono puede ser económicamente viable y constituir una situación de doble ganancia.

\section{LA RETENCIÓN DE CARBONO EN EL SUELO}

Los tiempos largos de recambio de carbono en el suelo son favorecidos por materiales deficientes en nutrientes y ricos en lignina y ceras, inundaciones, bajas temperaturas, texturas arcillosas, alto nivel de bases, agregación y superficies de cargas variables. Los tiempos cortos de recambio son favorecidos por materiales ricos en nutrientes y carbohidratos, aireación, altas temperaturas, texturas arenosas, acidez y superficies de cargas bajas (Zapata, 2006). La magnitud con la cual un suelo puede ser un reservorio continuo de carbono depende del balance entre las tasas de los procesos de adquisición y de degradación, tanto del carbono residente como del recientemente adquirido, poco se conoce acerca de estos 
procesos, especialmente en los suelos ácidos de las sabanas tropicales (Fisher et al., 1998).

Fisher et al., (1994) reportaron que las gramíneas africanas introducidas en las sabanas de Colombia acumulan carbono orgánico en el suelo, esto fue obtenido en pastos de Andropogon gayanus (Ag), Brachiaria humidicola (Bh) y Brachiaria dictyoneura $(\mathrm{Bd})$ en dos sitios de la altillanura colombiana y fueron comparados con sabanas nativas adyacentes. La distribución de carbono en el suelo a diferentes profundidades en los tres pastos introducidos, de los cuales uno tenía la leguminosa forrajera Arachis pintoi en asociación, donde fueron medidas grandes de carbono $\left(17.9 \mathrm{~kg} \mathrm{C} / \mathrm{m}^{2}\right.$ ) en comparación con las gramíneas introducidas y sabana nativa, las cuales adicionaron una cantidad total de COS, entre 0 y $80 \mathrm{~cm}$ de profundidad, de 12.6 y $14.7 \mathrm{~kg} \mathrm{C} / \mathrm{m}^{2}$ respectivamente. Es importante indicar que más del $75 \%$ del carbono adicional fue encontrado por debajo de la capa arable a $20 \mathrm{~cm}$ de profundidad, por lo tanto, Fisher et al., (1994) concluyeron que este carbono debería ser menos propenso a la oxidación y a la pérdida durante cualquier fase de cultivo que se llevara a cabo en sistemas integrados de cultivos y pastos, puesto que deberían ser capaces de soportar rotaciones con cultivos anuales (Figura 3).

El carbono acumulado en el suelo debe originarse del carbono fijado por el pasto, es decir, debe venir de la productividad primaria neta (PPN) del pasto. Por lo tanto, es apropiado determinar la PPN de los pastos de la altillanura Colombiana, que a diferencia de los cultivos anuales de ciclo corto que tienen un desarrollo sincronizado y pasan por etapas de crecimiento bien definidas, mientras los forrajes tienen un ciclo de iniciación continuo, crecimiento y muerte de unidades individuales (vástagos en gramíneas y ramas en leguminosas). A medida que el volumen acumulado del forraje en un pasto aumenta, la tasa de senescencia y mortalidad de las unidades más viejas aumenta hasta que finalmente se igualan con las tasas de iniciación de las nuevas unidades, lo que comúnmente se conoce como producción máxima. 


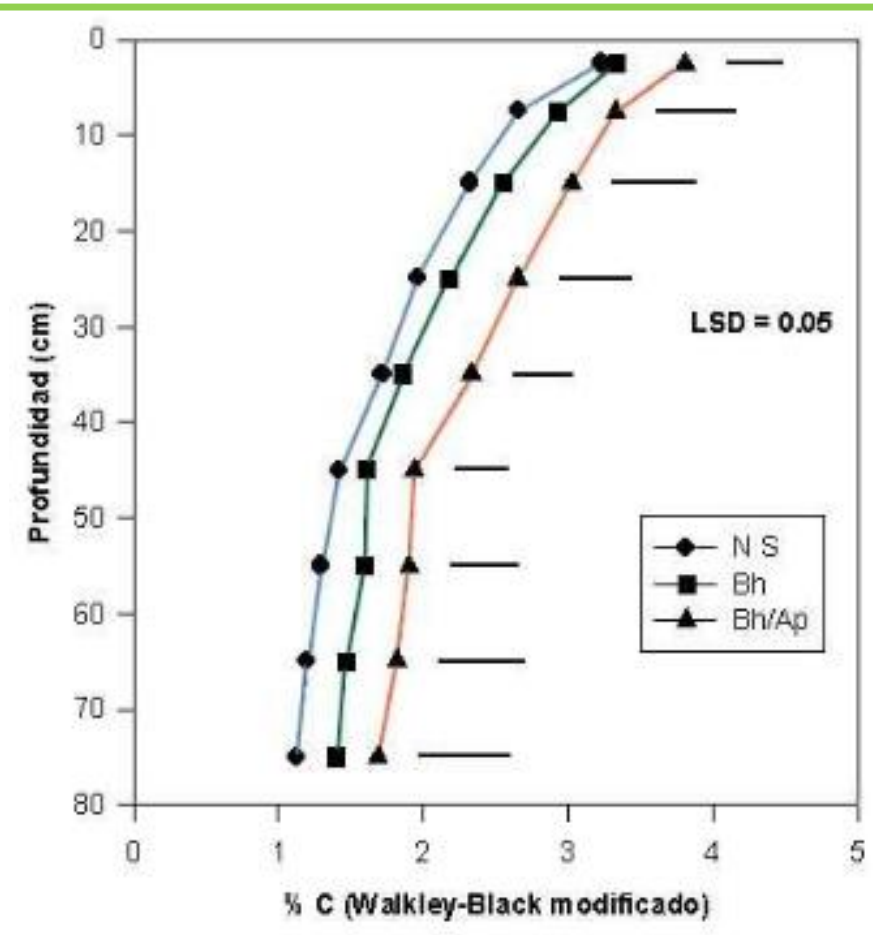

Figura 3. La distribución de $C$ orgánico en el suelo por profundidad por forrajes introducidos de la gramínea Brachiaria humidicola monocultivo (Bh) y asociada con la leguminosa Arachis pintoi (Bh/Ap) contrastado con la sabana nativa (NS) en el los llanos orientales de Colombia.

Fuente: Fisher et al., (1994)

Fisher et al., (1998) calcularon que la PPN aérea de Andropogon gayanus en Santander de Quilichao, Colombia, era de $17 \mathrm{~g} / \mathrm{m}^{2} / \mathrm{d}$ la cual continúa durante los 250 días de la etapa de crecimiento en Carimagua (precipitación anual $2.140 \mathrm{~mm}$ y altas temperaturas todo el año) la PPN de $A$. gayanus debería ser de $4.3 \mathrm{~kg} / \mathrm{m}$. En relación con la medición de la producción y longitud de raíces de pastos bajo pastoreo Rao et al., (1996) demostraron que el recambio de esta parte tiene la misma tasa que la del material aéreo, y aplicaron esto, para la máxima medida de producción de raíces. Además, Fisher et al., (1998) calcularon la cantidad anual de hojarasca y excrementos que retornan al suelo en $3.35-4.05 \mathrm{~kg} / \mathrm{m}^{2}$, dependiendo del nivel de utilización (usualmente no sobrepasa un 20 o 30\%) que equivale a 1.3-1.6 $\mathrm{kg} \mathrm{C} / \mathrm{m} / \mathrm{año}$, asumiendo que la materia seca tiene un $40 \%$ de carbono. También afirmaron que si la PPN de las raíces es la misma que la de la parte aérea, las entradas de carbono total en una gramínea o leguminosa bajo pastoreo están entre 2.6 a $3.2 \mathrm{~kg} \mathrm{C} / \mathrm{m}^{2} / \mathrm{año}$. 


\section{ANÁLISIS}

Las gramíneas responsables del secuestro de carbono medido por Fisher et al., (1994) son tan solo dos ( $A$. gayanus y $B$. humidicola), sin embargo, están representadas por pocos gramos de semilla y un número pequeño de plantas individuales, por esta razón es importante la estimación directa de su potencial para el secuestro del carbono en los suelos, pero, ¿cuáles serían los criterios para seleccionar plantas con la capacidad para secuestrar carbono?, ¿sería satisfactorio seleccionar especies con una alta PPN y al mismo tiempo con proporciones amplias de C:N en la hojarasca aérea y en las raíces?. No se pude afirmar nada con certeza hasta que no se conozca más acerca de los procesos.

Se podría pensar que en los pastos introducidos en las praderas tropicales generará un nuevo equilibrio de la MOS en 20 años, si esto es así, ¿cuál es el nuevo equilibrio y qué lo controlará?, ¿cuál será el efecto de las altas proporciones de C:N que Fisher et al., (1994) midieron en los pastos introducidos en las sabanas de Sudamérica?.

La razón por la que los pastos naturales son quemados es para que el forraje joven esté disponible más rápidamente para los animales en pastoreo. Aunque hay evidencias de que la ventaja es más aparente que real, ¿se podría convencer a los agricultores para que cambien sus prácticas tradicionales de manejo?, si se demostrara a los gobiernos que esto es cierto, ¿ayudaría a que sus compromisos en el marco de trabajo de la Convención sobre el Cambio Climático se logre?, ¿cuáles son las posibilidades para la negociación de servicios internacionales ecológicos y para que los agricultores sean subsidiados para adoptar prácticas de manejo que aumenten el secuestro de carbono en el suelo?

La PPN de los forrajes tropicales ha sido subestimada, al igual que el potencial de los pastos establecidos en el trópico para secuestrar carbono en el suelo, se ha identificado un número de problemas no resueltos que necesitan una mayor investigación. Además, hay problemas más amplios que requieren debatirse y pensarse mejor, por ejemplo, si los países no adscritos al protocolo de Kyoto 
quieren beneficiarse de los reservorios como el carbono del suelo, deberán presionar tan fuerte como sea posible para cambiar las definiciones del MDL en el Artículo 12 del PK.

Por lo anterior, con base en las revisiones realizadas y los análisis respectivos, se realizó una matriz DOFA, con el fin de identificar acciones viables que determinen la posibilidad de la captura de carbono en forrajes como una alternativa productiva para la altillanura colombiana el en el departamento del Meta (Tabla 1).

Tabla 1. Matriz DOFA, de la captura de carbono en forrajes como una alternativa productiva para la altillanura colombiana el en el departamento del Meta

\begin{tabular}{|c|c|}
\hline Debilidades & Oportunidades \\
\hline $\begin{array}{l}\text { Se tiene poco conocimiento en el Departamento } \\
\text { sobre la captura de carbono como una } \\
\text { alternativa productiva. }\end{array}$ & $\begin{array}{l}\text { Las condiciones agroecológicas y } \\
\text { socioeconómicas favorables de las pasturas en } \\
\text { la captura de carbono. }\end{array}$ \\
\hline $\begin{array}{l}\text { Falta de conocimiento en el comportamiento del } \\
\text { carbono en las condiciones de la altillanura. }\end{array}$ & $\begin{array}{l}\text { Las pasturas pueden ser un gran sumidero de } \\
\mathrm{CO}_{2} \text { a través de la acumulación de } \mathrm{C} \text { en la } \mathrm{MO} \\
\text { y en la biomasa viva de las plantas. }\end{array}$ \\
\hline $\begin{array}{l}\text { Poco apoyo económico por parte del estado a } \\
\text { la investigación e incentivos de nuevas } \\
\text { alternativas productivas. }\end{array}$ & $\begin{array}{l}\text { Constante generación de empleo durante el } \\
\text { establecimiento de las pasturas y su estimación } \\
\text { de carbono. }\end{array}$ \\
\hline $\begin{array}{l}\text { Ausencia de industria en la región capaz de } \\
\text { procesar la semilla sin deteriorar su calidad o } \\
\text { características organolépticas. }\end{array}$ & $\begin{array}{l}\text { Las distintitas industrias interesadas en la } \\
\text { captura de carbono como alternativa a la } \\
\text { mitigación del cambio climático }\end{array}$ \\
\hline Fortalezas & Amenazas \\
\hline $\begin{array}{l}\text { Sus características físico-químicas y contenidos } \\
\text { materia orgánica inigualables por las pasturas. }\end{array}$ & $\begin{array}{l}\text { El apoyo del gobierno a especies con potencial } \\
\text { para la elaboración de biocombustibles y no a } \\
\text { incentivar la implementación de alternativas q } \\
\text { contribuyan a la mitigación del cambio } \\
\text { climático. }\end{array}$ \\
\hline $\begin{array}{l}\text { El establecimiento de las praderas, lo que } \\
\text { genera constantes ingresos para los ganaderos }\end{array}$ & $\begin{array}{l}\text { Poca industria en la región capaz de } \\
\text { transformar y dar un valor agregado a las } \\
\text { pasturas establecidas sin deteriorar su calidad. }\end{array}$ \\
\hline $\begin{array}{l}\text { La alta producción ganadera con que se cuenta } \\
\text { en el departamento, permitiendo utilizar las } \\
\text { praderas como reservorios de carbono. }\end{array}$ & $\begin{array}{l}\text { Poca aceptación de por parte de la comunidad } \\
\text { de la altillanura debido al desconocimiento del } \\
\text { manejo, rentabilidad, beneficios, usos y } \\
\text { demanda de la captura de carbono a nivel } \\
\text { nacional y mundial }\end{array}$ \\
\hline
\end{tabular}




\section{CONCLUSIONES}

En sistemas pastoriles mixtos los pastos hacen uso del fertilizante residual aplicado a los cultivos, los cuales necesitan mayores niveles de fertilidad que los pastos, por lo tanto es el cultivo, no el pasto el que paga el costo económico y ambiental, en consecuencia los sistemas agropastoriles, que usan fertilizantes para aumentar la producción agrícola y la acumulación de carbono podrían ser económicamente viables al constituir una situación de doble ganancia.

La generación de forrajes adaptados a suelos marginales es clave para maximizar los beneficios de las pasturas mejoradas, además de incrementar el secuestro de carbono las pasturas pueden ser parte de sistemas de labranza mínima y de sistemas agrosilvopastoriles, con efectos positivos en el rendimiento de los cultivos y la mitigación del efecto invernadero. Existen vacíos de conocimiento sobre el mecanismo de captura a largo plazo de carbono especialmente en las capas profundas del suelo, además la introducción de árboles puede mejorar aún más el secuestro de carbono.

Las pasturas bien manejadas pueden acumular carbono de forma similar a los bosques especialmente cuando se incluyen árboles, por lo tanto las mejores oportunidades de mitigación están en la recuperación de las pasturas degradadas y el manejo de los suelos orgánicos.

\section{REFERENCIAS BIBLIOGRÁFICAS}

1. Amézquita E, Rao I, Rivera M, Corrales I, Bernal J (eds). Sistemas Agropastoriles: Un enfoque integrado para el manejo sostenible de oxisoles de los Llanos Orientales de Colombia. Centro Internacional de Agricultura Tropical (CIAT), Ministerio de Agricultura y Desarrollo Rural (MADR) de Colombia y Corporación Colombiana de Investigación Agropecuaria (Corpoica). Cali, Colombia, 288 p. 2013.

2. Arana, F. El mundo de la ecología. Editorial Océano. España. 2001.

3. Bravo, E. El sol bajo las patas de los caballos. Derechos de la naturaleza y geoingeniería, 2 p. 2011. Disponible En: http://www.grain.org/es/article/entries/4336el-sol-bajo-las-patas-de-los-caballos-derechos-de-la-naturaleza-y-geoingenieria

4. Buringh P, Dudal R. Agricultural land use in space and time. En: M.G. Wolman and F.G.A. Fournier (eds) Land transformations in agriculture. Scope 32, J. Wiley \& Sons Inc. Inglaterra. 1987.

5. Cárdenas E, Bustamante A, Espitia F, Páez A. Productividad en materia seca y captura de carbono en un sistema silvopastoril y un sistema tradicional en cinco 
fincas ganaderas de piedemonte en el departamento de Casanare. Red Med Vet, 24: 51-57. 2012.

6. Casanova F, Petit J, Solorio J. Los sistemas agroforestales como alternativa de la captura de carbono en el trópico mexicano. Rev. Chapingo, 17 (1): 133-143. 2011.

7. Céspedes F, Fernández J, Gobbi J, Bernardis A. Reservorio de carbono en suelo y raíces de un pastizal y una pradera bajo pastoreo. Revista Fitotecnia Mexicana, 35 (1): 79-86. 2012.

8. Ciesla WM. Cambio climático, bosques y ordenación forestal. Una visión de conjunto. Estudio FAO Montes N. 126. 147 p. 1996.

9. CORPOICA. Sistema de apoyo a la toma de decisiones para la selección de especies forrajeras (STDF), Versión 4.0. 19 p. 2014.

10. Davidson EA, Nepstad DC, Klink C, Trumbore SE. Pasture soils as carbon sink. Revista Nature, 376 (6540): 472-473. 1995.

11. Davila HA. Estimación de la cantidad de carbono almacenado en los sistemas agroforestales de cacao (Theobroma cacao L.) en los departamentos de Suchitepquez y Retalhuleu del suroccidente de Guatemala. Universidad de San Carlos de Guatemala, Facultad de Agronomía, 90 p. 2011.

12. Decaens T, Lavelle P, Jean J, Escobar G, Rippstein G. Impact of land management on soil macrofauna in the Oriental Llanos of Colombia. Rev Eur J Soil Biol. 30: 157168. 1994.

13. Euguren L. El mercado del carbono en América y el Caribe: balance y perspectivas. Naciones Unidas, CEPAL, División de Desarrollo Sostenible y Asentamientos Humanos, Santiago de Chile, 83 p. 2004.

14. Falkowski PG, Baber RT, Smetacek. Biochemical controls and feedbacks on ocean primary production, Science magazine, 281: 200. 1998. Disponible En: http://academic.engr.arizona.edu/HWR/Brooks/GC572-2004/readings/falkowski.pdf

15. FAO (Food Agricultural Organization). FAO yearbook-production. FAO Statistics Series Vol. 46, N. 112. Italia. 1993.

16. FAO (Food Agricultural Organization, Roma). Sistema de uso de la tierra en los trópicos húmedos y la emisión y secuestro de $\mathrm{CO}_{2}$. Informes sobre recursos mundiales de los suelos. 98 p. 2000.

17. FAO (Food Agricultural Organization, EMBRAPA). Protección de los recursos naturales en sistemas ganaderos: Los sistemas agroforestales pecuarios en América Latina. Consulta de expertos FAO. (18-22 septiembre de 2000. Juiz de Flora, MG, Brasil). 2001.

18. FAO (Food Agricultural Organization). Captura de carbono en los suelos para un mejor manejo de la tierra. Informes sobre recursos mundiales de suelos. Organización de las Naciones Unidas para la Agricultura y la Alimentación, Roma. Vol. 96.70 p. 2002.

19. Faría J. Manejo de pastos y forrajes en la ganadería doble propósito. X Seminario de pastos y forrajes, Maracaibo, Venezuela. 9 p. 2006.

20. Fisher MJ, Rao IM, Ayarza MA, Lascano CE, Sanz JI, Thomas RJ, Vera RR. Carbon storage by introduced deep-rooted grasses in the South American savannas. Revista Nature. 371: 236-238. 1994.

21. Fisher MJ, Thomas RJ, Rao IM. Management of tropical pastures in acid-soil savannas of South America for carbon sequestration in the soil. En: Management of Carbon Sequestration in Soil. Advances in Soil Science Series. Boca Raton, Florida, Estados Unidos, CRC Press, p. 405-420. 1998.

22. Gallardo JF, Merino A. El ciclo del carbono y la dinámica de los sistemas forestales. En: El papel de los bosques españoles en la mitigación del cambio climático. p 43-64. 2007. 
23. García J. Análisis del potencial de emisión de dióxido de carbono del páramo de Chingaza y lineamientos para su conservación en el contexto del mecanismo de desarrollo limpio. Tesis de Grado Ecólogo, Pontificia Universidad Javeriana, Facultad de Estudios Ambientales y Rurales, 110 p. 2003.

24. Greenland DJ. Land use and soil carbon in different agroecological zones. En: Soil management and greenhouse effect. Advances in Soil Science Series. Lewis Pubs. Estados Unidos. p 9-24. 1995.

25. Guggenberger G, Thomas RJ, Zech W. Soil organic matter within earthworm casts of an anecic-endogeic tropical pasture community. Publicación de Appl. Soil Ecol. 1996.

26. Hodgson J. Grazing Management: Science into Practice. Longman Scientific \& Technical. Inglaterra. 1990.

27. Houghton RA. Changes in the storage of terrestrial carbon since 1850. En: Soils and global change. Advances in Soil Science series. Lewis Pubs. Estados Unidos. 1995.

28. Intergovermental Panel on Climate Change (IPPC). Land use, land-use change, and forestry special report. Cambridge University Press, 377 p. 2000.

29. Intergovermental Panel on Climate Change (IPPC). Cambio climático y biodiversidad. Documento técnico V del IPCC, 85 p. 1995.

30. Keeling, C D. The influence of mauna loa observatory on the development of atmospheric $\mathrm{CO}_{2}$ research". In Mauna Loa Observatory: A 20 ${ }^{\text {th }}$ Anniversary Report. National Oceanic and Atmospheric Administration Special Report, September 1978, edited by John Miller, p 36-54. Boulder, CO: NOAA Environmental Research Laboratories. 1978.

31. Lavelle P, Gilot C, Fragoso C, Pashanasi B. Soil fauna and sustainable land use in the humid tropics. En: Soil resilience and sustainable land use. CAB International, Wallingford. 1994.

32. Lefévre N, Watson A, Olsen A, Rios AF, Pérez F, Johannessen T. A decrease in the sink for atmospheric $\mathrm{CO}_{2}$ in the North Atlantic, Geophysical Research Letters., 31, L07306, 2004. doi:10.1029/2003GL018957.

33. Long SP, Jones MB, Roberts MJ. Primary Productivity of Grass Ecosystems of the Tropics and Sub-tropics. Chapman and Hall. Estados Unidos. 1992.

34. Manley J.T., G.E. Schuman, J.D. Reeder, Hart R.H. Rangeland soil carbon and nitrogen responses to grazing. Revista J. Soil and Water Cons., 50: 294-298. 1995.

35. Martínez E, Fuentes JP, Acevedo EH. Carbono orgánico y propiedades del suelo. R.C. Cuelo Nutr. Veg., 8 (1): 68-96. 2008.

36. Mendieta M, Rocha LR. Sistemas Agroforestales. Universidad Nacional Agraria. Managua, Nicaragua. 115 p. 2007.

37. Mora, V. Fijación, emisión y balance de gases de efecto invernadero en pasturas en monocultivo y en sistemas silvopastoriles de fincas lecheras intensivas de las zonas altas de Costa Rica. Tesis Mag. Sc. Turrialba, Costa Rica. CATIE. 92 p. 2001.

38. Pearson CJ, Ison R. Agronomy of grassland systems. Cambridge University Press. Inglaterra. 1987.

39. PNUMA (Programa de las Naciones Unidas para el Medio Ambiente. GEO 5, Perspectivas de medio ambiente mundial. Editora Novo Art SA, Panama, 528 p. 2012.

40. Oades JM. The retention of organic matter in soils. Revista Biogeochem. 5: 35 -70. 1998.

41. Organización de Naciones Unidas. Protocolo de Kyoto de la convención marco de las naciones unidas sobre el cambio climático. 24 p. 1998.

42. Ortiz AG, Riascos LD. Almacenamiento y fijación de carbono del sistema agroforestal cacao Theobroma cacao L y Laurel Cordia alliodora (Ruiz \& Pavón) oken en la reserva indígena de Talamanca, Costa Rica. Trabajo de grado Ingeniero Agroforestal, 
Universidad de Nariño, Facultad de Ciencias Agrícolas, Programa de Ingeniería Agroforestal, 111 p. 2006.

43. Rao IM, Ayarza MA, Herrera P. Root distribution and production in native and introduced pastures in the South American savannas. En: Proceedings of the 5th Symposium of International Society of Root Research. Kluwer Academic Publishers. Holanda. 1996.

44. Rojas JP. La compensación de emisiones y los mercados de carbono. Éxito empresarial, $\quad 145$ : $1-4.2011 .2$ Disponible http://www.cegesti.org/exitoempresarial/publicaciones/publicacion 145140411 es.pd f

45. Scholes RJ, Hall DO. The carbon budget of tropical savannas, woodlands and grasslands. En: Global change: effect of coniferous forests and grasslands. SCOPE, 56: 69-100. 1995.

46. Scripps Institution of Oceonography y National Oceanic and Atmospheric Administration (NOAA). Monthly mean atmospheric carbon dioxide at Mauna Loa Observatory, Hawaii. 2014.

47. Secretaria de Medio Ambiente y Recursos Naturales (SEMARNAT). Cambio climático. Ciencia, evidencia y acciones. México, 81 p. 2009.

48. Spain J, Pereira JM, Gualdron R. A flexible grazing management system proposed for the advanced evaluation of associations of tropical grasses and legumes. Proc. $15^{\text {th }}$ Int. Grassl. Cong., Kyoto. Japón, 1995.

49. Szott L.; Ibrahim M.; Beer J. The hamburguer connection hangover: Cattle, pasture land degradation and alternative land use in Central America. Turrialba, CR, CATIE. 71 p. 2000.

50. Thomas RJ Asakawa NM. Decomposition of leaf litter from tropical forage grasses and legumes. Rev Soil Biol. Biochem, 25: 1351-1361. 1993.

51. Thomas RJ, Fisher MJ, Ayarza MA, Sanz JI. The role of forage grasses and legumes in maintaining the productivity of acid soils in Latin America. En: Soil Management: Experimental Basis for Sustainability and Environmental Quality. Advances in Soil Science Series. Lewis Pubs. Estados Unidos. 1995.

52. Thornely JHM, Fowler D., Cannell MGR. Terrestrial carbon storage resulting from $\mathrm{CO}_{2}$ and nitrogen fertilization in temperate grasslands. Rev PI. Cell Environ, 14 (9): 1007-1011. 1991.

53. Villanueva $\mathrm{C}$, Ibrahim M, Torres K, Torres M. Planificación agroecológica de fincas ganaderas: La experiencia de la subcuenca Copán, Honduras. Centro Agronómico Tropical de Investigación y Enseñanza (CATIE). Turrialba, Costa Rica, 36 p. 2008.

54. Zapata R. Química de los procesos pedogenéticos. Universidad Nacional de Colombia, Medellín. 358 p. 2006. 